doi: $10.1007 / \mathrm{s} 13366-014-0233-3$

\title{
HYPERBOLIC IS THE ONLY HILBERT GEOMETRY HAVING CIRCUMCENTER OR ORTHOCENTER GENERALLY
}

\author{
JÓZSEF KOZMA AND ÁRPÁD KURUSA
}

\begin{abstract}
A Hilbert geometry is hyperbolic if and only if the perpendicular bisectors or the altitudes of any trigon form a pencil. We also prove some interesting characterizations of the ellipse.
\end{abstract}

\section{InTRODUCTION}

Hilbert geometries, introduced by David Hilbert in 1899 [6], are natural generalizations of hyperbolic geometry, and hence the question immediately arises if some properties of a Hilbert geometry are specific to the hyperbolic geometry. For a recent survey on the results see [5].

To place our subject in a broader context we mention that it can also be considered as a so-called ellipsoid characterization problem in Euclidean space, which is often treated as characterization of Euclidean spaces (inner product spaces) among the normed spaces (See [1] and $[15,16]$ ). Further, the unitary imaginary unit sphere in generalized space-time model $[7,8]$ can also be considered as a Hilbert geometry.

In this article we prove two results: the existence of a circumcenter in every trigon (Theorem 5.1) or the existence of an orthocenter in every trigon (Theorem 5.2) renders Hilbert geometry hyperbolic. Moreover, we also prove two characterizations of ellipses in Section 4.

\section{Preliminaries}

Points of $\mathbb{R}^{n}$ are denoted as $\mathbf{a}, \mathbf{b}, \ldots$; the line through different points $\mathbf{a}$ and $\mathbf{b}$ is denoted by $\mathbf{a b}$, the open segment with endpoints $\mathbf{a}$ and $\mathbf{b}$ is denoted by $\overline{\mathbf{a b}}$. Non-degenerate triangles are called trigons.

For given different points $\mathbf{p}$ and $\mathbf{q}$ in $\mathbb{R}^{n}$, and points $\mathbf{x}, \mathbf{y} \in \mathbf{p q}$ one has the unique linear combinations $\mathbf{x}=\lambda_{1} \mathbf{p}+\mu_{1} \mathbf{q}, \mathbf{y}=\lambda_{2} \mathbf{p}+\mu_{2} \mathbf{q}\left(\lambda_{1}+\mu_{1}=1, \lambda_{2}+\mu_{2}=1\right)$ which allows to define the cross ratio

$$
(\mathbf{p}, \mathbf{q} ; \mathbf{x}, \mathbf{y})=\frac{\mu_{1} \lambda_{2}}{\lambda_{1} \mu_{2}},
$$

of the points $\mathbf{p}, \mathbf{q}, \mathbf{x}$ and $\mathbf{y}$, provided that $\lambda_{1} \mu_{2} \neq 0$ (see [2, page 243]).

1991 Mathematics Subject Classification. 53A35; 51M09, 52A20.

Key words and phrases. Hilbert geometry, hyperbolic geometry, circumcenter, orthocenter, ellipsoid characterization. 
Definition 2.1 ([2, page 297]). Let $\mathcal{H} \subset \mathbb{R}^{n}(n \geq 2)$ be an open and convex set with boundary $\partial \mathcal{H}$. The metric $d_{\mathcal{H}}: \mathcal{H} \times \mathcal{H} \rightarrow \mathbb{R}_{0 \leq}$ defined by

$$
d_{\mathcal{H}}(\mathbf{x}, \mathbf{y})= \begin{cases}\frac{1}{2}|\ln |(\mathbf{p}, \mathbf{q} ; \mathbf{x}, \mathbf{y})||, & \text { if } \mathbf{x} \neq \mathbf{y}, \text { where }\{\mathbf{p}, \mathbf{q}\}=\mathbf{x y} \cap \partial \mathcal{H}, \\ 0, & \text { if } \mathbf{x}=\mathbf{y},\end{cases}
$$

is called the Hilbert metric on $\mathcal{H}$. The pair $\left(\mathcal{H}, d_{\mathcal{H}}\right)$ is called Hilbert geometry.

Note that as all the defining conditions of a Hilbert geometry $\left(\mathcal{H}, d_{\mathcal{H}}\right)$ is projective invariant, two Hilbert geometries are isomorphic if there is a projective map between their sets of points. Further, the generalized Cayley-Klein model of the hyperbolic geometry $\mathbb{H}^{n}$ is, in fact, a special kind of Hilbert geometry $\left(\mathcal{E}, d_{\mathcal{E}}\right)$ given by an ellipsoid $\mathcal{E}$.

Let $\mathbf{a}, \mathbf{b}$ be different points in $\mathcal{H}$. For any $\mathbf{c} \in \mathcal{H} \cap(\mathbf{a b} \backslash\{\mathbf{b}\})$ the hyperbolic ratio $^{1}$ of the triple $\mathbf{a}, \mathbf{b}, \mathbf{c}$ is defined by

$$
\langle\mathbf{a}, \mathbf{b} ; \mathbf{c}\rangle_{\mathcal{H}}=\left\{\begin{aligned}
-\frac{\sinh d_{\mathcal{H}}(\mathbf{c}, \mathbf{a})}{\sinh d_{\mathcal{H}}(\mathbf{b}, \mathbf{c})}, & \text { if } \mathbf{c} \in \overline{\mathbf{a b}} \\
\frac{\sinh d_{\mathcal{H}}(\mathbf{c}, \mathbf{a})}{\sinh d_{\mathcal{H}}(\mathbf{b}, \mathbf{c})}, & \text { otherwise. }
\end{aligned}\right.
$$

Perpendicularity of $\mathcal{H}$-lines, non-empty intersections of Euclidean lines with $\mathcal{H}$, in Hilbert geometry is defined in [2, pp. 119-121] $]^{2}$. It is based on the notion of the foot of a point of $\mathcal{H}$ on an $\mathcal{H}$-line.

Let $\ell$ be an $\mathcal{H}$-line and let the point $\mathbf{g} \in \mathcal{H}$ be outside of $\ell$. The point $\mathbf{f} \in \ell$ is the $\ell$-foot of $\mathbf{g}$, if $d_{\mathcal{H}}(\mathbf{g}, \mathbf{x}) \geq d_{\mathcal{H}}(\mathbf{g}, \mathbf{f})$ for every $\mathbf{x} \in \ell$. $^{3}$

A line $\ell^{\prime}$ intersecting the line $\ell$ in a point $\mathbf{f}$ is said to be $\mathcal{H}$-perpendicular to $\ell$ if $\mathbf{f}$ is an $\ell$-foot of $\mathbf{g}$ for every $\mathbf{g} \in \ell^{\prime} \backslash\{\mathbf{f}\}$. We denote this relation by $\ell^{\prime} \perp_{\mathcal{H}} \ell$. $^{4}$

It is proved in $[2,(28.11)]$ that, if $\mathcal{H}$ is strictly convex, then for any given point $\mathbf{f} \in \mathcal{H}$ and $\mathcal{H}$-line $\ell$ there exists a unique $\mathcal{H}$-line $\ell^{\prime}$ such that it goes through $\mathbf{f}$ and $\ell^{\prime} \perp_{\mathcal{H}} \ell$. Moreover, the Euclidean line containing $\ell^{\prime}$ is the one that connects $\mathbf{f}$ and the intersection of those tangents of $\mathcal{H}$ that touch $\mathcal{H}$ at the points $\partial \ell$.

A set of lines is said to form a pencil if they have a common (maybe ideal) point. This point is called the center of the pencil. We say that a set of $\mathcal{H}$-lines forms a pencil with center $\mathbf{c}$, if the corresponding euclidean lines form a pencil with center $\mathbf{c}$. Thus the set of those lines that are $\mathcal{H}$-perpendicular to an arbitrary fixed line $\ell$ is a pencil.

Based on the foregoing, one can speak about the

- $\mathcal{H}$-perpendicular bisector of a segment $\overline{\mathbf{a b}}$, as the unique line through the midpoint of $\overline{\mathbf{a b}}$, that is $\mathcal{H}$-perpendicular to the line $\mathbf{a b}$, and the

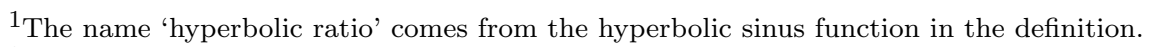

${ }^{2}$ In fact, it is defined for projective metrics.

${ }^{3}$ Observe that a point may have more $\ell$-foots in general.

${ }^{4}$ Notice, that $\perp_{\mathcal{H}}$ is not necessarily a symmetric relation. In fact it is symmetric if and only if $\mathcal{H}$ is an ellipse [10].
} 
- $\mathcal{H}$-altitude of a triangle $\triangle \mathbf{a b c}$, as a line through one of the vertices of $\triangle \mathbf{a b c}$, that is $\mathcal{H}$-perpendicular to the corresponding opposite edge of $\triangle \mathbf{a b c}$.

These definitions ${ }^{5}$ extend the respective notion of the perpendicular bisector of a segment and the altitude of a triangle, as defined in hyperbolic geometry.

From now on, we assume that $\mathcal{H}$ is strictly convex and has $C^{2}$ boundary.

\section{UTILITIES}

The useful notations $\mathbf{u}_{\tau}=(\cos \tau, \sin \tau)$ and $\mathbf{u}_{\tau}^{\perp}=(-\sin \tau, \cos \tau)$ are used all over this article. Also the following technical lemmas and the notations will be used in proving our main results.

Lemma 3.1 ([13, Lemma 2.3]). Let $\mathbf{a}, \mathbf{b}$ and $\mathbf{c}$ be collinear points in a Hilbert geometry $\mathcal{H}$, and let $\mathbf{a b} \cap \partial \mathcal{H}=\{\mathbf{p}, \mathbf{q}\}$, such that $\mathbf{a}$ separates $\mathbf{p}$ and $\mathbf{b}$. Set a Euclidean coordinate system on $\mathbf{a b}$ such that the coordinates of $\mathbf{p}$ and $\mathbf{a}$ are 0 and 1 , respectively. Let $q, b$ and $c$, with assumptions $q>b>1$ and $0<c<q$, be the coordinates of $\mathbf{q}, \mathbf{b}$ and $\mathbf{c}$, respectively, in this coordinate system. Then we have

$$
\left|\langle\mathbf{a}, \mathbf{b} ; \mathbf{c}\rangle_{\mathcal{H}}\right|=\frac{|c-b|}{|c-1| \sqrt{b}} \sqrt{1+\frac{b-1}{q-b}} .
$$

Lemma 3.2 ([2, Lemma 12.1, pp. 226]). A bounded open convex set $\mathcal{H}$ in $\mathbb{R}^{n}$ $(n \geq 2)$ is an ellipsoid if and only if every section of it by any 2-dimensional plane is an ellipse.

Lemma 3.3. Let $\mathcal{H}$ be a convex body in the plane. Then

(i) there exists an ellipse $\mathcal{E}$ circumscribed around $\mathcal{H}$ with at least three different contact points $\mathbf{e}_{1}, \mathbf{e}_{2}, \mathbf{e}_{3}$ lying in $\partial \mathcal{H} \cap \partial \mathcal{E}$ such that the closed triangle $\triangle \mathbf{e}_{1} \mathbf{e}_{2} \mathbf{e}_{3}$ contains the center $\mathbf{c}$ of $\mathcal{E}$, and

(ii) if $\mathcal{H} \not \equiv \mathcal{E}$, then these contact points can be chosen so that in every neighborhood of one of them $\partial \mathcal{H} \backslash \partial \mathcal{E} \neq \emptyset$.

Let $t_{1}, t_{2}, t_{3}$ be the common support lines at $\mathbf{e}_{1}, \mathbf{e}_{2}, \mathbf{e}_{3}$, respectively. Then

(iii) $\mathbf{c}$ is in the interior of $\triangle \mathbf{e}_{1} \mathbf{e}_{2} \mathbf{e}_{3}$ if and only if $t_{1}, t_{2}, t_{3}$ form a trigon with vertices $\mathbf{m}_{1}=t_{2} \cap t_{3}, \mathbf{m}_{2}=t_{3} \cap t_{1}$ and $\mathbf{m}_{3}=t_{1} \cap t_{2}$;

(iv) $\mathbf{c}$ is in one of the edges of $\triangle \mathbf{e}_{1} \mathbf{e}_{2} \mathbf{e}_{3}$, say $\mathbf{c} \in \overline{\mathbf{e}_{2} \mathbf{e}_{3}}$, if and only if $t_{1}, t_{2}, t_{3}$ form a half strip with vertices $\mathbf{m}_{2}=t_{1} \cap t_{3}, \mathbf{m}_{3}=t_{2} \cap t_{1}$ and the ideal point $\mathbf{m}_{1}=t_{2} \cap t_{3}$.

If $\mathbf{b}_{1}, \mathbf{b}_{2}, \mathbf{b}_{3}$ are the midpoints of the segments $\overline{\mathbf{e}_{2} \mathbf{e}_{3}}, \overline{\mathbf{e}_{3} \mathbf{e}_{1}}$ and $\overline{\mathbf{e}_{1} \mathbf{e}_{2}}$, respectively, then

(v) the straight lines $\mathbf{m}_{i} \mathbf{b}_{i}(i=1,2,3)$ meet in $\mathbf{c}$.

\footnotetext{
${ }^{5}$ Notice that these notions could also be introduced by using $\perp_{\mathcal{H}}$ in the reverse order.
} 
Proof. Take the unique minimal area ellipse $\mathcal{E}$ containing $\mathcal{H}$ and let the center c of $\mathcal{E}$ be the origin $\mathbf{o}$.

(i) By $[3$, Theorem 2 (ii)] there is an integer $(5 \geq) m \geq 3$ such that there are contact points $\mathbf{e}_{1}, \ldots, \mathbf{e}_{m}$ lying in $\partial \mathcal{H} \cap \partial \mathcal{E}$ such that a positive linear combination of the contact points vanishes. This means that the origin is in the convex hull of these contact points, hence a (closed) trigon of three of them, say $\triangle \mathbf{e}_{1} \mathbf{e}_{2} \mathbf{e}_{3}$, also contains the origin.

(ii) Transform the configuration given in (i) with a linear affinity $\mu$ so that $\mathcal{D}=\mu(\mathcal{E})$ is the unit disc centered to o. Let $\mathbf{e}_{i}^{\prime}=\mu\left(\mathbf{e}_{i}\right)(i=1,2,3)$ and $\mathcal{H}^{\prime}=\mu(\mathcal{H})$.

By (i) the center $\mathbf{o}$ is in the trigon $\triangle \mathbf{e}_{1}^{\prime} \mathbf{e}_{2}^{\prime} \mathbf{e}_{3}^{\prime}$. Let $\varepsilon_{i} \in(-\pi, \pi]$ be such that $\mathbf{e}_{i}^{\prime}=$ $\mathbf{u}_{\varepsilon_{i}}$ and let the support function of $\mathcal{H}^{\prime}$ be denoted by $h_{\mathcal{H}^{\prime}}$. Define $\alpha_{i}:=\lim \sup \{\alpha$ : $\left.h_{\mathcal{H}^{\prime}}\left(\left[\varepsilon_{i}, \varepsilon_{i}+\alpha\right]\right)=\{1\}\right\}(i=1,2,3)$. If $\alpha_{i}$ is infinite, then $\mathcal{H} \equiv \mathcal{E}$, that is excluded. Assume that $\alpha_{k}=\min _{i=1,2,3} \alpha_{i}$ for some $k \in\{1,2,3\}$. Set $\mathbf{f}_{i}=\mu^{-1}\left(\mathbf{u}_{\varepsilon_{i}+\alpha_{k}}\right)$ $(i=1,2,3)$. Then $\mathbf{f}_{1}, \mathbf{f}_{2}, \mathbf{f}_{3}$ are contact points of $\partial \mathcal{H}$ and $\partial \mathcal{E}$, the trigon $\mathbf{f}_{1}, \mathbf{f}_{2}, \mathbf{f}_{3}$ contains the center $\mathbf{o}$, and in every neighborhood $\mathcal{N}$ of $\mathbf{f}_{k}(\partial \mathcal{H} \backslash \partial \mathcal{E}) \cap \mathcal{N} \neq \emptyset$.

(iii) and (iv) are easy consequences of the strict convexity of the ellipse $\mathcal{E}$.

(v) This readily follows if one transforms the ellipse into a circle by a linear affinity.

Lemma 3.4. For a small $\varepsilon>0$ let $\mathbf{r}, \mathbf{p}:(-\varepsilon, 0] \rightarrow \mathbb{R}^{2}$ be twice differentiable convex curves such that $\mathbf{p}(\tau)=p(\tau) \mathbf{u}_{\tau}$ and $\mathbf{r}(\tau)=r(\tau) \mathbf{u}_{\tau}$, where $p, r:(-\varepsilon, 0] \rightarrow \mathbb{R}_{+}$, $\lambda(\tau):=r(\tau) / p(\tau)$ takes its minimum value 1 at $\tau=0$, and $\max _{(-\delta, 0]} \lambda>1$ for every $\delta \in(0, \varepsilon)$.

Let $\tau_{n}$ be a sequence in $(-\varepsilon, 0]$ tending to 0 such that $\lambda\left(\tau_{n}\right)>1$ for every $n \in \mathbb{N}$. Then the tangent lines of $\mathbf{r}$ and $\mathbf{p}$ at $\mathbf{r}\left(\tau_{n}\right)$ and $\mathbf{p}\left(\tau_{n}\right)$, respectively, intersect each other in a point $\mathbf{m}\left(\tau_{n}\right)$ that tends to $\mathbf{p}(0)$ as $\tau_{n} \rightarrow 0$ so that it is on the same side of the line $\mathbf{0 p}\left(\tau_{n}\right)$ as $\mathbf{p}(0)$ is.

Proof. First we prove the statement with the assumption that $\lambda$ takes its minimum value 1 uniquely at $\tau=0$. This means that $\dot{\lambda}(0)=0, \ddot{\lambda}(0)>0$ and we have to prove that

the tangent lines of $\mathbf{r}$ and $\mathbf{p}$ at $\mathbf{r}(\tau)$ and $\mathbf{p}(\tau)$, respectively, intersect each other in a point $\mathbf{m}(\tau)$ that tends to $\mathbf{p}(0)$ as $\tau \rightarrow 0$ so that it is on the same side of the line $\mathbf{0 p}(\tau)$ as $\mathbf{p}(0)$ is.

Since $\dot{\mathbf{r}}=\lambda \dot{\mathbf{p}}+\dot{\lambda} \mathbf{p}, \dot{\mathbf{p}} \| \dot{\mathbf{r}}$ if and only if $\dot{\lambda}=0$, therefore $\mathbf{m}(\tau)$ exists uniquely for every $\tau \neq 0$. 


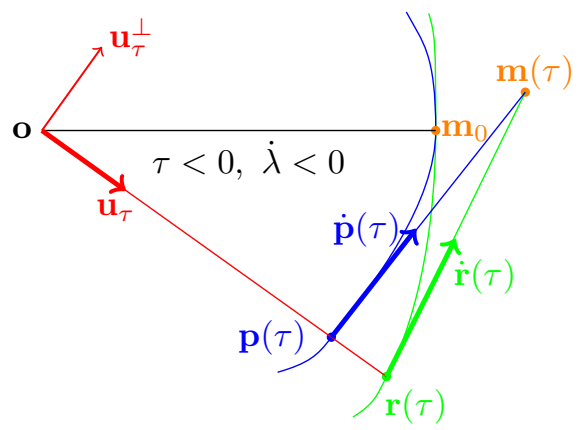

Figure 3.1. The crossing of the tangent lines.

We clearly have

$$
\pm|\mathbf{m}-\mathbf{p}| \frac{\dot{\mathbf{p}}}{|\dot{\mathbf{p}}|}+\mathbf{p}=\mathbf{m}= \pm|\mathbf{m}-\mathbf{r}| \frac{\dot{\mathbf{r}}}{|\dot{\mathbf{r}}|}+\mathbf{r}
$$

that is $\pm|\mathbf{m}-\mathbf{p}\|\dot{\mathbf{r}}|\dot{\mathbf{p}}+| \dot{\mathbf{r}}\| \dot{\mathbf{p}}| \mathbf{p}= \pm|\mathbf{m}-\mathbf{r}\|\dot{\mathbf{p}}|\dot{\mathbf{r}}+| \dot{\mathbf{p}}\| \dot{\mathbf{r}}| \mathbf{r}$.

Since $\dot{\mathbf{p}}=\dot{p} \mathbf{u}_{\tau}+p \mathbf{u}_{\tau}^{\perp}, \dot{\mathbf{r}}=\dot{r} \mathbf{u}_{\tau}+r \mathbf{u}_{\tau}^{\perp}$ and $\mathbf{u}_{\tau} \perp \mathbf{u}_{\tau}^{\perp}$, we obtain

$$
|\mathbf{m}-\mathbf{p}||\dot{\mathbf{r}}| p=|\mathbf{m}-\mathbf{r}||\dot{\mathbf{p}}| r
$$

and

$$
\pm|\mathbf{m}-\mathbf{p}\|\dot{\mathbf{r}}|\dot{p}+p| \dot{\mathbf{p}}\| \dot{\mathbf{r}}|= \pm|\mathbf{m}-\mathbf{r}\|\dot{\mathbf{p}}|\dot{r}+r| \dot{\mathbf{r}}\| \dot{\mathbf{p}}| .
$$

Multiplying (3.5) by $p$ then substituting (3.4) into the product results in

$$
\pm|\mathbf{m}-\mathbf{r}||\dot{\mathbf{p}}| r \dot{p}+p^{2}|\dot{\mathbf{p}}||\dot{\mathbf{r}}|= \pm|\mathbf{m}-\mathbf{r} \| \dot{\mathbf{p}}| p \dot{r}+p r|\dot{\mathbf{r}}||\dot{\mathbf{p}}|
$$

hence

$$
\pm|\mathbf{m}-\mathbf{r}|=\frac{p|\dot{\mathbf{r}}|(r-p)}{r \dot{p}-p \dot{r}}=\frac{|\dot{\mathbf{r}}| p^{2}(\lambda-1)}{\lambda p \dot{p}-p(\dot{\lambda} p+\lambda \dot{p})}=|\dot{\mathbf{r}}| \frac{\lambda-1}{-\dot{\lambda}} .
$$

This implies $\lim _{\tau \rightarrow 0}|\mathbf{m}(\tau)-\mathbf{r}(\tau)|=0$ via l'Hôspital's rule.

On the other hand, using (3.4) and putting (3.6) into (3.3) gives

$$
\frac{\lambda(\lambda-1)}{-\dot{\lambda}} \dot{\mathbf{p}}+\mathbf{p}=\mathbf{m}=\frac{\lambda-1}{-\dot{\lambda}} \dot{\mathbf{r}}+\mathbf{r} .
$$

As $\lambda \geq 1$, this implies that $\mathbf{m}$ is on the same side of $\mathbf{0 r}$ and $\mathbf{0 p}$ as $\mathbf{m}(0)=\mathbf{r}(0)=$ $\mathbf{p}(0)$. This proves claim (3.2).

For the proof of the statement in the lemma we take the broken line $\overline{\mathbf{q}}$ with vertices $\mathbf{p}\left(\tau_{n}\right)$ and edges $\overline{\mathbf{p}\left(\tau_{n}\right) \mathbf{p}\left(\tau_{n+1}\right)}$. It is clearly convex and can easily be deformed into a twice differentiable convex curve $\mathbf{q}$ so that $\mathbf{q}\left(\tau_{n}\right)=\mathbf{p}\left(\tau_{n}\right), \dot{\mathbf{q}}\left(\tau_{n}\right)=\dot{\mathbf{p}}\left(\tau_{n}\right)$ and $r(\tau) /|\mathbf{q}(\tau)|$ takes its minimum value 1 uniquely at $\tau=0$. Using claim (3.2) for $\mathbf{q}$ and $\mathbf{r}$ therefore immediately implies the lemma. 


\section{Characterizations of Ellipses}

The following configuration, construction, theorems, and the notations they introduce, are used in the next sections, but are interesting on their own too.

Definition 4.1. If a strictly convex body $\mathcal{H}$ is given in the plane, and the points $\mathbf{e}_{1}, \mathbf{e}_{2}, \mathbf{e}_{3}$ are placed on its border $\partial \mathcal{H}$, then the following configuration is defined.

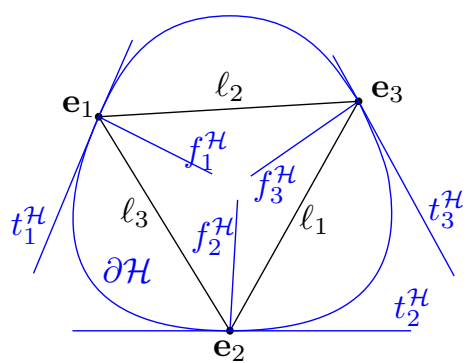

Figure 4.1. Construction for Definition 4.1

For every $i=1,2,3, \ell_{i}$ denotes the line $\mathbf{e}_{j} \mathbf{e}_{k}, t_{i}^{\mathcal{H}}$ denotes the tangent line of $\mathcal{H}$ at the point $\mathbf{e}_{i}$, and $f_{i}^{\mathcal{H}}$ denotes the straight line through $\mathbf{e}_{i}$ that forms a harmonic pencil with the lines $\ell_{j}, \ell_{k}, t_{i}^{\mathcal{H}}$, where $\{i, j, k\}=\{1,2,3\}$.

Theorem 4.2. ${ }^{6}$ Take a configuration given in Definition 4.1.

(i) For any ellipse $\mathcal{E}$ the lines $f_{1}^{\mathcal{E}}, f_{2}^{\mathcal{E}}, f_{3}^{\mathcal{E}}$ form a pencil.

(ii) If the lines $f_{1}^{\mathcal{H}}, f_{2}^{\mathcal{H}}, f_{3}^{\mathcal{H}}$ form a pencil for any points $\mathbf{e}_{1}, \mathbf{e}_{2}, \mathbf{e}_{3} \in \partial \mathcal{H}$, then $\mathcal{H}$ is an ellipse.

Proof. First note that not only keep projectivities the cross ratio, but takes any tangent line of a curve into a tangent line of the image curve.

\footnotetext{
${ }^{6}$ After this theorem was proved it turned out, that the dual of this statement is, via the theorems of Menelaus and Ceva equivalent to Segre's result in [17, §3] which, as noted in [12, 6.15. Tétel], does not use the finiteness of the geometry but only the commutativity of the field; note that following [11, p. 133], the perspectivity of the circimscribed and inscribed triangle was named as $\pi$-property in [12].
} 


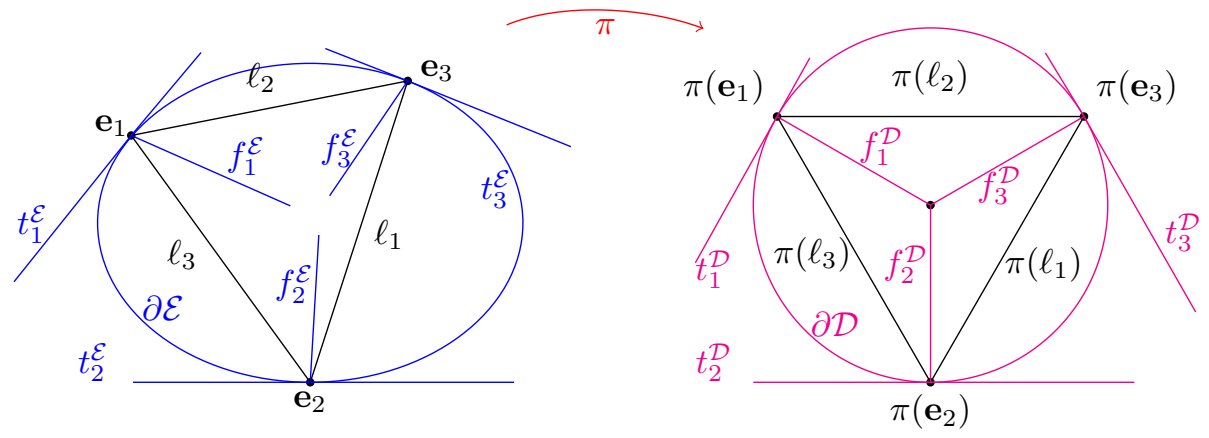

Figure 4.2. Transforming the ellipse $\mathcal{E}$ into a disc $\mathcal{D}$ and the triangle $\triangle \mathbf{e}_{1} \mathbf{e}_{2} \mathbf{e}_{3}$ into a regular one.

(i) Taking a suitable affinity we may assume that ellipse $\mathcal{H}$ is a disc $\mathcal{D}$. The projective group is three-transitive ${ }^{7}$ on every conic, hence we may assume that $\mathbf{e}_{1}, \mathbf{e}_{2}, \mathbf{e}_{3}$ forms a regular triangle on the circle $\partial \mathcal{D}$. Then, obviously, the lines $f_{1}, f_{2}, f_{3}$ meet in the center of $\mathcal{C}$ that proves statement (i).

(ii) The condition remains unchanged if the configuration is transformed by a projective map, therefore we may assume that the points $\mathbf{e}_{1}, \mathbf{e}_{2}$ and $\mathbf{e}_{3}$ and $\mathbf{f}:=f_{1}^{\mathcal{H}} \cap f_{2}^{\mathcal{H}} \cap f_{3}^{\mathcal{H}}$ are such that $\mathbf{e}_{1}=(0,1), \mathbf{e}_{2}=(0,-1), \mathbf{e}_{3}=(1,0)$ and $\mathbf{f}=(\sqrt{2}-1,0)$.

Then, the straight lines $f_{1}^{\mathcal{H}}, f_{2}^{\mathcal{H}}$ and $f_{3}^{\mathcal{H}}$ are determined and from the conditions $-1=\left(\ell_{1}, \ell_{2} ; t_{3}^{\mathcal{H}}, f_{3}^{\mathcal{H}}\right)=\left(\ell_{2}, \ell_{3} ; t_{1}^{\mathcal{H}}, f_{1}^{\mathcal{H}}\right)=\left(\ell_{3}, \ell_{1} ; t_{2}^{\mathcal{H}}, f_{2}^{\mathcal{H}}\right)$, we get the equations $y=1, y=-1$ and $x=1$ for $t_{1}^{\mathcal{H}}, t_{2}^{\mathcal{H}}$ and $t_{3}^{\mathcal{H}}$, respectively.

Now choose a general point $\mathbf{h} \in \partial \mathcal{H}$ different from $\mathbf{e}_{1}, \mathbf{e}_{2}$, and let $\partial \mathcal{E}_{\mathbf{h}}$ be the unique ellipse through the points $\mathbf{e}_{1}, \mathbf{e}_{2}, \mathbf{h}$ with tangents $t_{1}^{\mathcal{E}}:=t_{1}^{\mathcal{H}}$ and $t_{2}^{\mathcal{E}}:=t_{2}^{\mathcal{H}}$ at $\mathbf{e}_{1}$ and $\mathbf{e}_{2}$, respectively.

Let us introduce some new notations (See Figure 4.3.):

- $t_{\mathbf{h}}^{\mathcal{H}}$ is the tangent of $\mathcal{H}$ at $\mathbf{h}$;

- $\overline{\ell_{i}}$ is the line $\mathbf{h e}_{i}$ for $i=1,2 ; \overline{\ell_{3}}$ is the line $\mathbf{e}_{1} \mathbf{e}_{2}$;

- $f_{i}^{\mathcal{H}}$ is the line through $\mathbf{e}_{i}$ for $i=1,2$ such that $-1=\left(\bar{\ell}_{j}, \bar{\ell}_{k} ; t_{i}^{\mathcal{H}}, f_{i}^{\mathcal{H}}\right)$, where $\{i, j, k\}=\{1,2,3\}$

- $f_{\mathbf{h}}^{\mathcal{H}}$ is the line through $\mathbf{h}$ such that $-1=\left(\bar{\ell}_{1}, \bar{\ell}_{2} ; t_{\mathbf{h}}^{\mathcal{H}}, f_{\mathbf{h}}^{\mathcal{H}}\right)$.

We denote the analogous objects for the ellipse $\mathcal{E}_{\mathbf{h}}$ in the same way except that the superscript $\mathcal{H}$ is exchanged to $\mathcal{E}$.

\footnotetext{
${ }^{7}$ This is easy to prove by using conic involutions.
} 


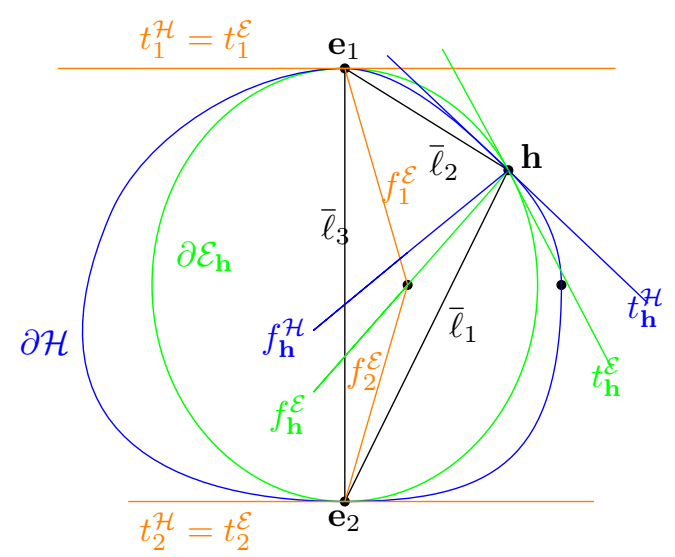

FiguRE 4.3. Introducing harmonic pencils

Since $t_{1}^{\mathcal{E}}=t_{1}^{\mathcal{H}}$ and $t_{2}^{\mathcal{E}}=t_{2}^{\mathcal{H}}$ we clearly have $f_{i}^{\mathcal{H}}=f_{i}^{\mathcal{E}}$ for $i=1,2$.

As the lines $f_{1}^{\mathcal{E}}, f_{2}^{\mathcal{E}}$ and $f_{\mathbf{h}}^{\mathcal{E}}$ form a pencil by (i), and $f_{1}^{\mathcal{H}}, f_{2}^{\mathcal{H}}$ and $f_{\mathbf{h}}^{\mathcal{H}}$ form a pencil by the condition in (ii), we deduce that the lines $f_{\mathbf{h}}^{\mathcal{H}}$ and $f_{\mathbf{h}}^{\mathcal{E}}$ intersect each other not only in $\mathbf{h}$, but also in $f_{1}^{\mathcal{H}} \cap f_{2}^{\mathcal{H}}=f_{1}^{\mathcal{E}} \cap f_{2}^{\mathcal{E}}$, hence they coincide.

Thus, we have $t_{\mathbf{h}}^{\mathcal{H}}=t_{\mathbf{h}}^{\mathcal{E}}$. So it makes sense to introduce the notations $t_{i}:=t_{i}^{\mathcal{E}}=$ $t_{i}^{\mathcal{H}}$ for $i=1,2$ and $t_{\mathbf{h}}:=t_{\mathbf{h}}^{\mathcal{E}}=t_{\mathbf{h}}^{\mathcal{H}}$.

Let $r:(-\pi, \pi] \rightarrow \mathbb{R}_{+}$be such that $\mathbf{h}(\varphi)=r(\varphi) \mathbf{u}_{\varphi}$ is in $\partial \mathcal{H}$, for every $\varphi \in(-\pi, \pi]$.

Then a tangent vector of $\partial \mathcal{H}$ at $\mathbf{h}(\varphi)$ is $\dot{\mathbf{h}}(\varphi)=\dot{r}(\varphi) \mathbf{u}_{\varphi}+r(\varphi) \mathbf{u}_{\varphi}^{\perp}$ which is parallel to the tangent of the unique ellipse $\partial \mathcal{E}_{\mathbf{h}(\varphi)}$.

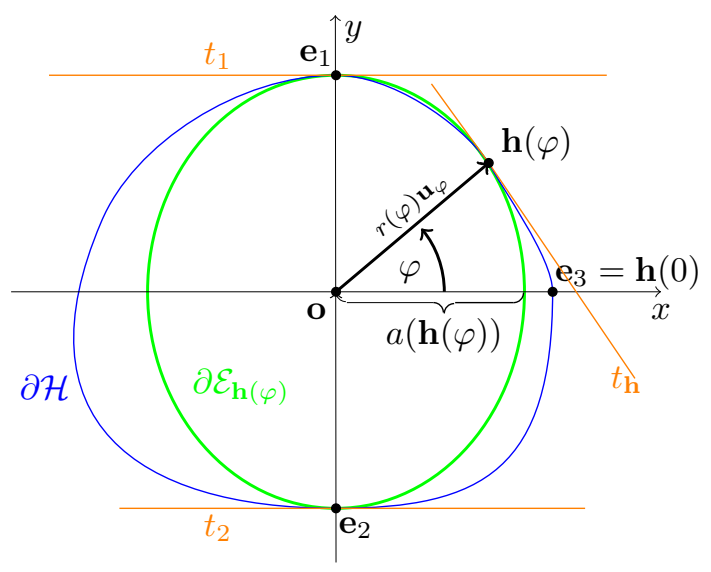

Figure 4.4. Parametrization of $\partial \mathcal{H}$ 
The ellipse $\partial \mathcal{E}_{\mathbf{h}(\varphi)}$ goes through the points $\mathbf{e}_{1}, \mathbf{e}_{2}, \mathbf{h}(\varphi)$ and it has tangents $t_{1}$ and $t_{2}$ at $\mathbf{e}_{1}$ and $\mathbf{e}_{2}$, respectively, therefore its equation is $\frac{x^{2}}{a^{2}}+y^{2}=1$ for some $a=a(\mathbf{h}(\varphi))$. Putting the coordinate of $\mathbf{h}(\varphi)$ into this equation we get

$$
1=r^{2}(\varphi)\left(\frac{\cos ^{2} \varphi}{a^{2}}+\sin ^{2} \varphi\right), \text { that is, } a^{2}=\frac{r^{2}(\varphi) \cos ^{2} \varphi}{1-r^{2}(\varphi) \sin ^{2} \varphi} .
$$

On the other hand, the slope of the tangent of the ellipse at $(x, y)$ is $\frac{d y}{d x}=\frac{-x}{y a^{2}}$ which at the point $\mathbf{h}(\varphi)$ is

$$
\frac{\dot{r}(\varphi) \sin \varphi+r(\varphi) \cos \varphi}{\dot{r}(\varphi) \cos \varphi-r(\varphi) \sin \varphi}=\frac{d y}{d x}=\frac{-x}{y a^{2}}=\frac{-\cos \varphi}{a^{2} \sin \varphi} .
$$

This implies

$$
\frac{\dot{r}(\varphi)}{r(\varphi)}=\frac{\left(1-a^{2}\right) \sin \varphi \cos \varphi}{a^{2} \sin ^{2} \varphi+\cos ^{2} \varphi}=\frac{\left(1-\frac{r^{2}(\varphi) \cos ^{2} \varphi}{1-r^{2}(\varphi) \sin ^{2} \varphi}\right) \sin \varphi \cos \varphi}{\frac{r^{2}(\varphi) \cos ^{2} \varphi}{1-r^{2}(\varphi) \sin ^{2} \varphi} \sin ^{2} \varphi+\cos ^{2} \varphi}=\left(1-r^{2}(\varphi)\right) \tan \varphi
$$

At every $\varphi$, where $r(\varphi) \neq 1$, this gives

$$
\frac{\dot{r}(\varphi)}{r(\varphi)\left(1-r^{2}(\varphi)\right)}=\tan \varphi
$$

which, by integration, yields

$$
\frac{-1}{2} \ln \frac{\left|1-r^{2}(\varphi)\right|}{r^{2}(\varphi)}=-\ln |\cos \varphi|+c_{0}
$$

for a constant $c_{0}$. An equivalent reformulation of this is

$$
r(\varphi)=\frac{1}{\sqrt{1 \pm c_{1} \cos ^{2} \varphi}},
$$

where $c_{1}$ is a constant. Substituting this into (4.1), $a^{2}\left(1 \pm c_{1}\right)=1$ follows, hence $a$ is the same constant for all ellipses $\partial \mathcal{E}_{\mathbf{h}(\varphi)}$, which are therefore a fixed ellipse $\partial \mathcal{E}$. This means that $\partial \mathcal{H}$ is a subset of $\partial \mathcal{E}$ having equation $\left(1 \pm c_{1}\right) \cdot x^{2}+y^{2}=1$.

However, $\partial \mathcal{H}$ contains the point $\mathbf{e}_{3}=(1,0)$ too, hence $c_{1}=0$ and therefore $\partial \mathcal{H}$ is the unit circle centered at the origin. This proves statement (ii).

Definition 4.3. Take a configuration according to Definition 4.1. We construct a set of geometric object in the following way: Chose a point $\mathbf{x}_{i}$ close to $\mathbf{e}_{i}$ on the open segment $\sigma_{i}=\overline{\mathbf{e}_{j} \mathbf{e}_{k}}$ for every $i=1,2,3$, where $\{j, k\}=\{1,2,3\} \backslash\{i\}$.

Let the lines $\mathbf{e}_{2} \mathbf{x}_{3}, \mathbf{e}_{3} \mathbf{x}_{1}$ and $\mathbf{e}_{1} \mathbf{x}_{2}$ be denoted by $\ell_{1}^{\prime}, \ell_{2}^{\prime}, \ell_{3}^{\prime}$, respectively.

Take the points $\mathbf{v}_{1}=\ell_{2}^{\prime} \cap \ell_{3}^{\prime}, \mathbf{v}_{2}=\ell_{3}^{\prime} \cap \ell_{1}^{\prime}, \mathbf{v}_{3}=\ell_{1}^{\prime} \cap \ell_{2}^{\prime}$, and denote the open segments $\overline{\mathbf{v}_{2} \mathbf{v}_{3}}, \overline{\mathbf{v}_{3} \mathbf{v}_{1}}$ and $\overline{\mathbf{v}_{1} \mathbf{v}_{2}}$, by $\sigma_{1}^{\prime}, \sigma_{2}^{\prime}$ and $\sigma_{3}^{\prime}$, respectively.

Further, we take the points $\mathbf{x}_{1}^{t}=t_{1} \cap \ell_{2}^{\prime}, \mathbf{x}_{2}^{t}=t_{2} \cap \ell_{3}^{\prime}, \mathbf{x}_{3}^{t}=t_{3} \cap \ell_{1}^{\prime}$, and $\mathbf{x}_{1}^{\mathcal{H}}=\partial \mathcal{H} \cap\left(\ell_{2}^{\prime} \backslash\left\{\mathbf{e}_{3}\right\}\right), \mathbf{x}_{2}^{\mathcal{H}}=\partial \mathcal{H} \cap\left(\ell_{3}^{\prime} \backslash\left\{\mathbf{e}_{1}\right\}\right), \mathbf{x}_{3}^{\mathcal{H}}=\partial \mathcal{H} \cap\left(\ell_{1}^{\prime} \backslash\left\{\mathbf{e}_{2}\right\}\right)$. These points of intersection do exist if $\mathbf{x}_{i}$ are chosen close enough to $\mathbf{e}_{i}(i=1,2,3)$. 


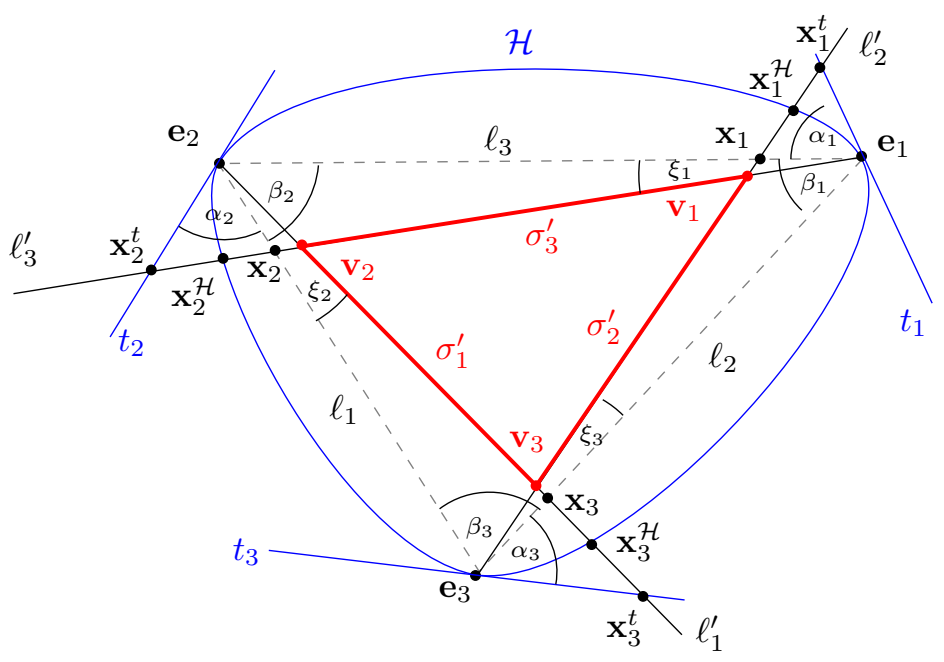

Figure 4.5. A construction for the triple asymptotic $\mathcal{H}$-triangle.

Finally, let the magnitude of the angles $\angle \mathbf{x}_{2} \mathbf{e}_{1} \mathbf{e}_{2}, \angle \mathbf{x}_{3} \mathbf{e}_{2} \mathbf{e}_{3}, \angle \mathbf{x}_{1} \mathbf{e}_{3} \mathbf{e}_{1}$ be denoted by $\xi_{1}, \xi_{2}, \xi_{3}$, respectively, that of the angles $\angle \mathbf{x}_{1}^{t} \mathbf{e}_{1} \mathbf{e}_{2}, \angle \mathbf{x}_{2}^{t} \mathbf{e}_{2} \mathbf{e}_{3}, \angle \mathbf{x}_{3}^{t} \mathbf{e}_{3} \mathbf{e}_{1}$ be denoted by $\alpha_{1}, \alpha_{2}, \alpha_{3}$, respectively, and that of the angles $\angle \mathbf{e}_{3} \mathbf{e}_{1} \mathbf{e}_{2}, \angle \mathbf{e}_{1} \mathbf{e}_{2} \mathbf{e}_{3}, \angle \mathbf{e}_{2} \mathbf{e}_{3} \mathbf{e}_{1}$ be denoted by $\beta_{1}, \beta_{2}, \beta_{3}$, respectively.

Theorem 4.4. Take a construction according to definition 4.3. For every $i=$ $1,2,3$ denote the Euclidean midpoint of the segment $\sigma_{i}$ by $\mathbf{b}_{i}$ and the $\mathcal{H}$-midpoint of the segment $\sigma_{i}^{\prime}$ by $\mathbf{b}_{i}^{\mathcal{H}}$

The lines $f_{1}^{\mathcal{H}}, f_{2}^{\mathcal{H}}, f_{3}^{\mathcal{H}}$ form a pencil if and only if the points $\mathbf{x}_{1}, \mathbf{x}_{2}$ and $\mathbf{x}_{3}$ can be chosen for any $\varepsilon, \delta>0$ so that

$$
\begin{array}{r}
\left|\mathbf{b}_{1}^{\mathcal{H}}-\mathbf{b}_{1}\right|+\left|\mathbf{b}_{2}^{\mathcal{H}}-\mathbf{b}_{2}\right|+\left|\mathbf{b}_{3}^{\mathcal{H}}-\mathbf{b}_{3}\right|<\varepsilon, \\
\left|\mathbf{x}_{1}-\mathbf{e}_{1}\right|+\left|\mathbf{x}_{2}-\mathbf{e}_{2}\right|+\left|\mathbf{x}_{3}-\mathbf{e}_{3}\right|<\delta .
\end{array}
$$

Proof. Since $d_{\mathcal{H}}\left(\mathbf{v}_{j}, \mathbf{b}_{i}^{\mathcal{H}}\right)=d_{\mathcal{H}}\left(\mathbf{b}_{i}^{\mathcal{H}}, \mathbf{v}_{k}\right)$, where $\{i, j, k\}=\{1,2,3\}$, (2.2) implies $\left(\mathbf{e}_{j}, \mathbf{x}_{k}^{\mathcal{H}} ; \mathbf{v}_{j}, \mathbf{b}_{i}^{\mathcal{H}}\right)=\left(\mathbf{e}_{j}, \mathbf{x}_{k}^{\mathcal{H}} ; \mathbf{b}_{i}^{\mathcal{H}}, \mathbf{v}_{k}\right)$, hence

$$
\begin{aligned}
1 & =\frac{\left(\mathbf{e}_{j}, \mathbf{x}_{k}^{\mathcal{H}} ; \mathbf{v}_{j}, \mathbf{b}_{i}^{\mathcal{H}}\right)}{\left(\mathbf{e}_{j}, \mathbf{x}_{k}^{\mathcal{H}} ; \mathbf{b}_{i}^{\mathcal{H}}, \mathbf{v}_{k}\right)}=\frac{\left(\mathbf{e}_{j}, \mathbf{x}_{k}^{\mathcal{H}}, \mathbf{v}_{j}\right) /\left(\mathbf{e}_{j}, \mathbf{x}_{k}^{\mathcal{H}}, \mathbf{b}_{i}^{\mathcal{H}}\right)}{\left(\mathbf{e}_{j}, \mathbf{x}_{k}^{\mathcal{H}} ; \mathbf{b}_{i}^{\mathcal{H}}\right) /\left(\mathbf{e}_{j}, \mathbf{x}_{k}^{\mathcal{H}}, \mathbf{v}_{k}\right)} \\
& =\frac{\left(\mathbf{e}_{j}, \mathbf{x}_{k}^{\mathcal{H}}, \mathbf{v}_{k}\right)\left(\mathbf{e}_{j}, \mathbf{x}_{k}^{\mathcal{H}}, \mathbf{v}_{j}\right)}{\left(\mathbf{e}_{j}, \mathbf{x}_{k}^{\mathcal{H}} ; \mathbf{b}_{i}^{\mathcal{H}}\right)^{2}}=\frac{\left|\mathbf{e}_{j}-\mathbf{v}_{k}\right|\left|\mathbf{e}_{j}-\mathbf{v}_{j}\right|}{\left|\mathbf{x}_{k}^{\mathcal{H}}-\mathbf{v}_{k}\right|\left|\mathbf{x}_{k}^{\mathcal{H}}-\mathbf{v}_{j}\right|} \frac{1}{\left(\mathbf{e}_{j}, \mathbf{x}_{k}^{\mathcal{H}} ; \mathbf{b}_{i}^{\mathcal{H}}\right)^{2}} .
\end{aligned}
$$

From now on, assume that $\xi_{i} \rightarrow 0$ for every $i=1,2,3$. Then $\mathbf{x}_{k}^{\mathcal{H}} \rightarrow \mathbf{e}_{k}$, hence the affine midpoint of $\overline{\mathbf{e}_{j} \mathbf{x}_{k}^{\mathcal{H}}}$ converges to $\mathbf{b}_{i}$, and therefore $\mathbf{b}_{i}^{\mathcal{H}} \rightarrow \mathbf{b}_{i}$ if and only if $\left(\mathbf{e}_{j}, \mathbf{x}_{k}^{\mathcal{H}} ; \mathbf{b}_{i}^{\mathcal{H}}\right) \rightarrow 1$. 


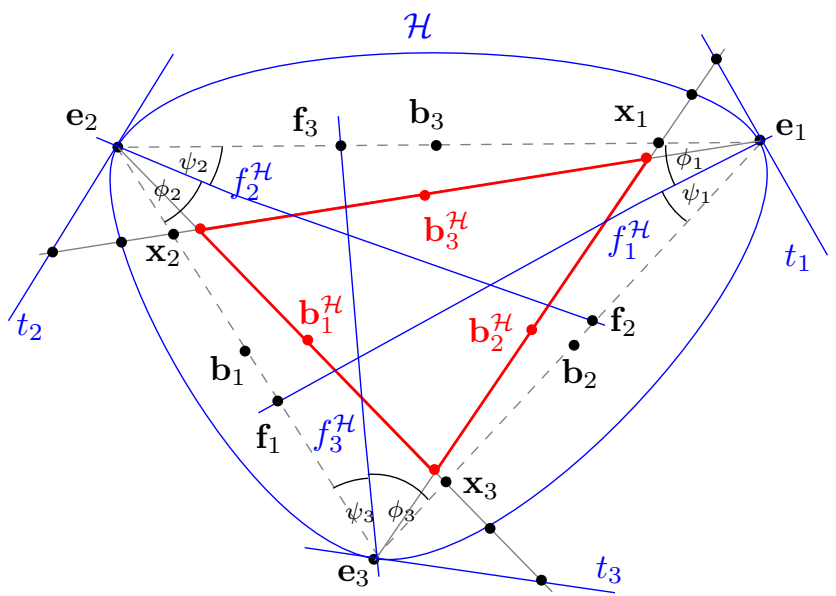

FigurE 4.6. The construction with the midpoints.

As we have $\frac{\left|\mathbf{e}_{j}-\mathbf{v}_{k}\right|}{\left|\mathbf{x}_{k}^{\mathcal{H}}-\mathbf{v}_{j}\right|} \rightarrow 1$, (4.2) implies the asymptotic equation

$$
\left(\mathbf{e}_{j}, \mathbf{x}_{k}^{\mathcal{H}} ; \mathbf{b}_{i}^{\mathcal{H}}\right)^{2} \sim \frac{\left|\mathbf{e}_{j}-\mathbf{v}_{j}\right|}{\left|\mathbf{x}_{k}^{\mathcal{H}}-\mathbf{v}_{k}\right|}
$$

which, in the light of the previous reasoning, means that

$$
\mathbf{b}_{i}^{\mathcal{H}} \rightarrow \mathbf{b}_{i} \text { if and only if }\left|\mathbf{e}_{j}-\mathbf{v}_{j}\right| \sim\left|\mathbf{x}_{k}^{\mathcal{H}}-\mathbf{v}_{k}\right| \text {. }
$$

Using the law of sines we obtain

$$
\begin{aligned}
& \frac{\left|\mathbf{x}_{k}^{\mathcal{H}}-\mathbf{v}_{k}\right|}{\left|\mathbf{e}_{j}-\mathbf{v}_{j}\right|} \\
= & \frac{\left|\mathbf{x}_{k}^{t}-\mathbf{e}_{j}\right|-\left|\mathbf{v}_{k}-\mathbf{e}_{j}\right|-\left|\mathbf{x}_{k}^{\mathcal{H}}-\mathbf{x}_{k}^{t}\right|}{\left|\mathbf{e}_{i}-\mathbf{e}_{j}\right| \sin \xi_{i} / \sin \left(\beta_{j}-\xi_{j}+\xi_{i}\right)} \\
= & \frac{\frac{\left|\mathbf{e}_{j}-\mathbf{e}_{k}\right|}{\sin \left(\beta_{k}+\alpha_{k}+\xi_{j}\right)} \sin \left(\beta_{k}+\alpha_{k}\right)-\frac{\left|\mathbf{e}_{j}-\mathbf{e}_{k}\right|}{\sin \left(\beta_{k}-\xi_{k}+\xi_{j}\right)} \sin \left(\beta_{k}-\xi_{k}\right)-\left|\mathbf{x}_{k}^{\mathcal{H}}-\mathbf{x}_{k}^{t}\right|}{\frac{\left|\mathbf{e}_{i}-\mathbf{e}_{j}\right|}{\sin \left(\beta_{j}-\xi_{j}+\xi_{i}\right)} \sin \xi_{i}} \\
= & \frac{\sin \left(\beta_{j}-\xi_{j}+\xi_{i}\right)}{\sin \xi_{i}} \frac{\left|\mathbf{e}_{j}-\mathbf{e}_{k}\right|}{\left|\mathbf{e}_{i}-\mathbf{e}_{j}\right|} \times \\
= & \left.\frac{\sin \left(\beta_{j}-\xi_{j}+\xi_{i}\right)}{\sin \left(\beta_{k}+\alpha_{k}\right)} \frac{\left|\sigma_{i}\right|}{\left|\sigma_{k}\right|} \times \frac{\sin \left(\beta_{k}-\xi_{k}\right)}{\sin \xi_{i}}-\frac{\left|\mathbf{x}_{k}^{\mathcal{H}}-\mathbf{x}_{k}^{t}\right|}{\left|\mathbf{e}_{j}-\mathbf{e}_{k}\right|}\right) \\
& \times\left(\frac{\tan \left(\beta_{k}+\alpha_{k}+\xi_{j}\right)}{\sin \xi_{j}+\cos \xi_{j} \tan \left(\beta_{k}+\alpha_{k}\right)}-\frac{\tan \left(\beta_{k}-\xi_{k}\right)}{\sin \xi_{j}+\cos \xi_{j} \tan \left(\beta_{k}-\xi_{k}\right)}-\frac{\left|\mathbf{x}_{k}^{\mathcal{H}}-\mathbf{x}_{k}^{t}\right|}{\left|\mathbf{e}_{j}-\mathbf{e}_{k}\right|}\right)
\end{aligned}
$$




$$
\begin{aligned}
= & \frac{\sin \left(\beta_{j}-\xi_{j}+\xi_{i}\right)}{\sin \xi_{i}} \frac{\left|\sigma_{i}\right|}{\left|\sigma_{k}\right|} \times \\
& \times\left(\frac{\sin \xi_{j}\left(\tan \left(\beta_{k}+\alpha_{k}\right)-\tan \left(\beta_{k}-\xi_{k}\right)\right)}{\left(\sin \xi_{j}+\cos \xi_{j} \tan \left(\beta_{k}+\alpha_{k}\right)\right)\left(\sin \xi_{j}+\cos \xi_{j} \tan \left(\beta_{k}-\xi_{k}\right)\right)}-\frac{\left|\mathbf{x}_{k}^{\mathcal{H}}-\mathbf{x}_{k}^{t}\right|}{\left|\mathbf{e}_{j}-\mathbf{e}_{k}\right|}\right) \\
= & \frac{\sin \xi_{j}}{\sin \xi_{i}} \frac{\left|\sigma_{i}\right|}{\left|\sigma_{k}\right|} \times \\
& \times\left(\frac{\sin \left(\beta_{j}-\xi_{j}+\xi_{i}\right)\left(\tan \left(\beta_{k}+\alpha_{k}\right)-\tan \left(\beta_{k}-\xi_{k}\right)\right)}{\left(\sin \xi_{j}+\cos \xi_{j} \tan \left(\beta_{k}+\alpha_{k}\right)\right)\left(\sin \xi_{j}+\cos \xi_{j} \tan \left(\beta_{k}-\xi_{k}\right)\right)}-\right. \\
\sim & \left.\frac{\sin \xi_{j}}{\sin \xi_{i}} \frac{\left|\sigma_{i}\right|}{\left|\sigma_{k}\right|} \frac{\sin \left(\beta_{j}-\xi_{j}+\xi_{i}\right)\left|\mathbf{x}_{k}^{\mathcal{H}}-\mathbf{x}_{k}^{t}\right|}{\tan \left(\beta_{k}+\alpha_{k}\right) \tan \beta_{k}}\right)
\end{aligned}
$$

Putting this into (4.3) results in

$$
\begin{aligned}
& \mathbf{b}_{1}^{\mathcal{H}} \rightarrow \mathbf{b}_{i} \Longleftrightarrow \frac{\sin \xi_{1}}{\sin \xi_{2}} \sim \frac{\left|\sigma_{1}\right|}{\left|\sigma_{3}\right|} \frac{\sin \beta_{2} \sin \alpha_{3}}{\sin \left(\beta_{3}+\alpha_{3}\right) \sin \beta_{3}}, \\
& \mathbf{b}_{2}^{\mathcal{H}} \rightarrow \mathbf{b}_{2} \Longleftrightarrow \frac{\sin \xi_{2}}{\sin \xi_{3}} \sim \frac{\left|\sigma_{2}\right|}{\left|\sigma_{1}\right|} \frac{\sin \beta_{3} \sin \alpha_{1}}{\sin \left(\beta_{1}+\alpha_{1}\right) \sin \beta_{1}}, \\
& \mathbf{b}_{3}^{\mathcal{H}} \rightarrow \mathbf{b}_{3} \Longleftrightarrow \frac{\sin \xi_{3}}{\sin \xi_{1}} \sim \frac{\left|\sigma_{3}\right|}{\left|\sigma_{2}\right|} \frac{\sin \beta_{1} \sin \alpha_{2}}{\sin \left(\beta_{2}+\alpha_{2}\right) \sin \beta_{2}} .
\end{aligned}
$$

Having arbitrary $\xi_{2} \rightarrow 0, \xi_{1} \rightarrow 0$ and $\xi_{3} \rightarrow 0$ can be so chosen, that the first two of these asymptotic equations are satisfied, and the fulfillment of the third one then depends exactly on their product. Such angles $\xi_{1}, \xi_{2}, \xi_{3}$ can be chosen therefore if and only if

$$
\sin \alpha_{1} \sin \alpha_{2} \sin \alpha_{3}=\sin \left(\beta_{1}+\alpha_{1}\right) \sin \left(\beta_{2}+\alpha_{2}\right) \sin \left(\beta_{3}+\alpha_{3}\right) .
$$

Denote the magnitude of the angles $\angle f_{1}^{\mathcal{H}} \ell_{2}, \angle f_{2}^{\mathcal{H}} \ell_{3}, \angle f_{3}^{\mathcal{H}} \ell_{1}$ by $\psi_{1}, \psi_{2}, \psi_{3}$, respectively, and the angles $\angle f_{1}^{\mathcal{H}} \ell_{3}, \angle f_{2}^{\mathcal{H}} \ell_{1}, \angle f_{3}^{\mathcal{H}} \ell_{2}$ by $\phi_{1}, \phi_{2}, \phi_{3}$, respectively (see Figure 4.6). We clearly have $\psi_{i}+\phi_{i}=\beta_{i}$ for every $i=1,2,3$.

Observe that

$$
\begin{aligned}
& -1=\left(\ell_{3}, \ell_{2} ; t_{1}, f_{1}^{\mathcal{H}}\right)=\frac{-\sin \alpha_{1} / \sin \left(\beta_{1}+\alpha_{1}\right)}{\sin \phi_{1} / \sin \psi_{1}} \\
& -1=\left(\ell_{1}, \ell_{3} ; t_{2}, f_{2}^{\mathcal{H}}\right)=\frac{-\sin \alpha_{2} / \sin \left(\beta_{2}+\alpha_{2}\right)}{\sin \phi_{2} / \sin \psi_{2}} \\
& -1=\left(\ell_{2}, \ell_{1} ; t_{3}, f_{3}^{\mathcal{H}}\right)=\frac{-\sin \alpha_{3} / \sin \left(\beta_{3}+\alpha_{3}\right)}{\sin \phi_{3} / \sin \psi_{3}}
\end{aligned}
$$

hence (4.4) is equivalent to

$$
1=\frac{\sin \phi_{1}}{\sin \psi_{1}} \frac{\sin \phi_{2}}{\sin \psi_{2}} \frac{\sin \phi_{3}}{\sin \psi_{3}} .
$$


Let $f_{i}^{\mathcal{H}} \cap \sigma_{i}=\mathbf{f}_{i}$ for every $i=1,2,3$. Then the law of sines gives

$$
\left(\mathbf{e}_{1}, \mathbf{e}_{2} ; \mathbf{f}_{3}\right)=\frac{\left|\sigma_{2}\right|}{\left|\sigma_{1}\right|} \frac{\sin \phi_{3}}{\sin \psi_{3}},\left(\mathbf{e}_{2}, \mathbf{e}_{3} ; \mathbf{f}_{1}\right)=\frac{\left|\sigma_{3}\right|}{\left|\sigma_{2}\right|} \frac{\sin \phi_{1}}{\sin \psi_{1}}, \text { and }\left(\mathbf{e}_{3}, \mathbf{e}_{1} ; \mathbf{f}_{2}\right)=\frac{\left|\sigma_{1}\right|}{\left|\sigma_{3}\right|} \frac{\sin \phi_{2}}{\sin \psi_{2}} .
$$

By Ceva's theorem the product of these ratios equals to 1 if and only if the lines $f_{1}^{\mathcal{H}}, f_{2}^{\mathcal{H}}$ and $f_{3}^{\mathcal{H}}$ form a pencil. This proves the theorem.

\section{Circumcenter and orthocenter in Hilbert geometry}

Existence of the circumcenter of a trigon, the common point of the three perpendicular bisectors, is a well known property in Euclidean plane. It can be formulated also for the hyperbolic plane [14, p. 350]: In hyperbolic geometry the perpendicular bisectors of any trigon form a pencil.

Theorem 5.1. If the $\mathcal{H}$-perpendicular bisectors of any trigon in the Hilbert geometry $\mathcal{H}$ form a pencil, then $\left(\mathcal{H}, d_{\mathcal{H}}\right)$ is the hyperbolic geometry.

Proof. We need to show that $\mathcal{H}$ is an ellipsoid. By Lemma 3.2 we only need to work in the plane, therefore from now on in this proof $\mathcal{H}$ is in a plane $\mathcal{P}$.

Suppose that $\mathcal{H}$ is not an ellipse. We shall have to arrive at a contradiction.

By (i) of Lemma 3.3 there exists an ellipse $\mathcal{E}$ circumscribed around $\mathcal{H}$ with at least three different contact points $\mathbf{e}_{1}, \mathbf{e}_{2}, \mathbf{e}_{3}$ lying in $\partial \mathcal{H} \cap \partial \mathcal{E}$ such that the closed triangle $\triangle \mathbf{e}_{1} \mathbf{e}_{2} \mathbf{e}_{3}$ contains the origin.

Suppose we have the configuration described in (iv) of Lemma 3.3.

Choose a plane $\mathcal{P}^{\prime}$ such that one of its open halfspace $\mathcal{S}$ contains $t_{1}$ and $\mathcal{E}$. Now choose a point $P$ out of $\mathcal{P}^{\prime} \cup \mathcal{P} \cup \mathcal{S}$. Let $\pi$ be the perspective projection of $\mathcal{P}$ into $\mathcal{P}^{\prime}$ through the point $P$. This projection $\pi$ clearly maps the configuration in $\mathcal{P}$ into a configuration in $\mathcal{P}^{\prime}$ that is described in (iii) of Lemma 3.3. Thus, since the statement of the theorem is of projective nature, it is enough to validate it for configurations described in (iii) of Lemma 3.3.

Take a construction defined by Definition 4.3 (see Figure 5.1), and let $\varepsilon=$ $\left|\mathbf{x}_{1}-\mathbf{e}_{1}\right|+\left|\mathbf{x}_{2}-\mathbf{e}_{2}\right|+\left|\mathbf{x}_{3}-\mathbf{e}_{3}\right|$.

By (v) of Lemma 3.3 the straight lines $\mathbf{m}_{i} \mathbf{b}_{i}(i=1,2,3)$ meet in the center $\mathbf{o}$ of the ellipse $\mathcal{E}$, which is in the interior of the trigon $\triangle \mathbf{e}_{1} \mathbf{e}_{2} \mathbf{e}_{3}$, therefore the center $\mathbf{o}$ of the ellipse $\mathcal{E}$ is in the interior of the trigon $\Delta \mathbf{v}_{1} \mathbf{v}_{2} \mathbf{v}_{3}$ too, if $\varepsilon$ is small enough, which we assume from now on.

According to Lemma 3.1 the lines $\ell_{1}^{\prime}, \ell_{2}^{\prime}, \ell_{3}^{\prime}$ contain the points

$$
\begin{aligned}
& \mathbf{e}_{2} \prec \mathbf{v}_{2} \prec \mathbf{b}_{1}^{\mathcal{H}} \preceq \mathbf{b}_{1}^{\mathcal{E}} \prec \mathbf{v}_{3} \prec \mathbf{x}_{3} \prec \mathbf{x}_{3}^{\mathcal{H}} \preceq \mathbf{x}_{3}^{\mathcal{E}}, \\
& \mathbf{e}_{3} \prec \mathbf{v}_{3} \prec \mathbf{b}_{2}^{\mathcal{H}} \preceq \mathbf{b}_{2}^{\mathcal{E}} \prec \mathbf{v}_{1} \prec \mathbf{x}_{1} \prec \mathbf{x}_{1}^{\mathcal{H}} \preceq \mathbf{x}_{1}^{\mathcal{E}}, \\
& \mathbf{e}_{1} \prec \mathbf{v}_{1} \prec \mathbf{b}_{3}^{\mathcal{H}} \preceq \mathbf{b}_{3}^{\mathcal{E}} \prec \mathbf{v}_{2} \prec \mathbf{x}_{2} \prec \mathbf{x}_{2}^{\mathcal{H}} \preceq \mathbf{x}_{2}^{\mathcal{E}},
\end{aligned}
$$

respectively, in the given order (see Figure 5.1). 


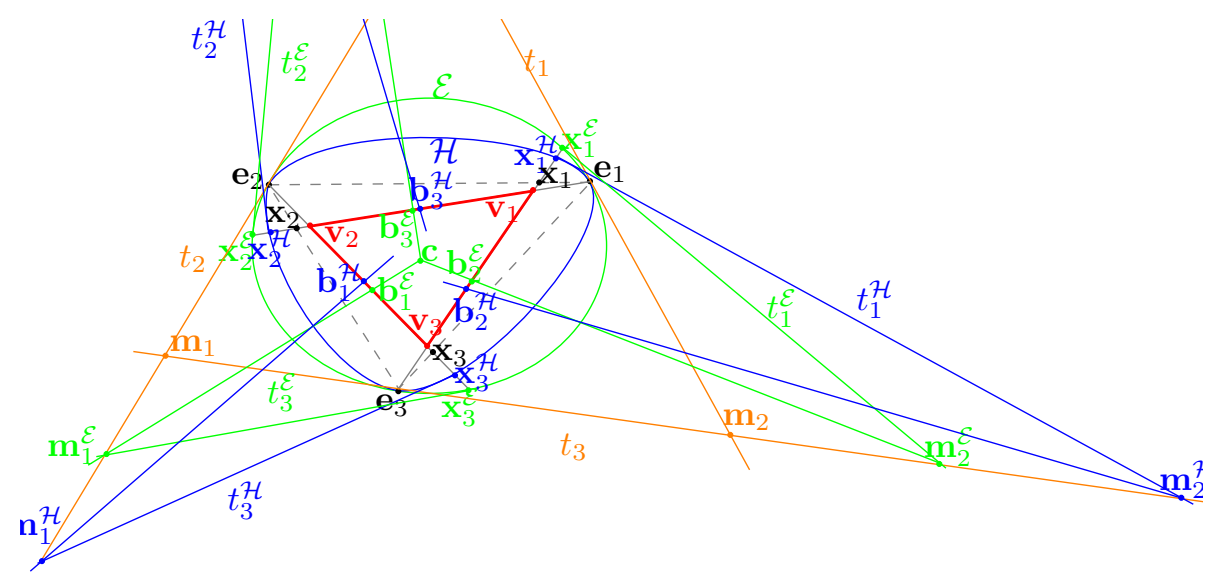

Figure 5.1. Constructions of circumcenters in $\mathcal{H}$ and $\mathcal{E}$.

Take the tangent lines $t_{i}^{\mathcal{H}}$ and $t_{i}^{\mathcal{E}}$ of $\mathcal{H}$ and $\mathcal{E}$ at the points $\mathbf{x}_{i}^{\mathcal{H}}$ and $\mathbf{x}_{i}^{\mathcal{E}}$, respectively, for every $i \in\{1,2,3\}$. Let $\mathbf{m}_{1}^{\mathcal{H}}=t_{2}^{\mathcal{H}} \cap t_{3}, \mathbf{m}_{2}^{\mathcal{H}}=t_{3}^{\mathcal{H}} \cap t_{1}, \mathbf{m}_{3}^{\mathcal{H}}=t_{1}^{\mathcal{H}} \cap t_{2}$, and $\mathbf{m}_{1}^{\mathcal{E}}=t_{2}^{\mathcal{E}} \cap t_{3}, \mathbf{m}_{2}^{\mathcal{E}}=t_{3}^{\mathcal{E}} \cap t_{1}$ and $\mathbf{m}_{3}^{\mathcal{E}}=t_{1}^{\mathcal{E}} \cap t_{2}$ (see Figure 5.1).

According to Lemma 3.4, the tangents $t_{1}, t_{2}, t_{3}$ contain the points

$$
\begin{gathered}
\mathbf{m}_{2} \prec \mathbf{e}_{1} \prec \mathbf{m}_{3} \prec \mathbf{m}_{3}^{\mathcal{E}} \preceq \mathbf{m}_{3}^{\mathcal{H}}, \quad \mathbf{m}_{3} \prec \mathbf{e}_{2} \prec \mathbf{m}_{1} \prec \mathbf{m}_{1}^{\mathcal{E}} \preceq \mathbf{m}_{1}^{\mathcal{H}}, \\
\mathbf{m}_{1} \prec \mathbf{e}_{3} \prec \mathbf{m}_{2} \prec \mathbf{m}_{2}^{\mathcal{E}} \preceq \mathbf{m}_{2}^{\mathcal{H}},
\end{gathered}
$$

in the given order, respectively (see Figure 5.1).

Notice, that for well-chosen $\mathbf{x}_{1}, \mathbf{x}_{2}, \mathbf{x}_{3}$ we have $\mathbf{x}_{i}^{\mathcal{H}} \neq \mathbf{x}_{i}^{\mathcal{E}}$ for some $i \in\{1,2,3\}$, say $\mathbf{x}_{1}^{\mathcal{H}} \neq \mathbf{x}_{1}^{\mathcal{E}}$, and then we also have $\mathbf{m}_{3}^{\mathcal{E}} \neq \mathbf{m}_{3}^{\mathcal{H}}$ and $\mathbf{b}_{2}^{\mathcal{E}} \neq \mathbf{b}_{2}^{\mathcal{H}}$ by (ii) of Lemma 3.3.

While the triangle $\triangle \mathbf{v}_{1} \mathbf{v}_{2} \mathbf{v}_{3}$ is in Int $\mathcal{H} \cap \operatorname{Int} \mathcal{E}$, letting $\varepsilon$ tend to 0 , it tends to the triangle $\triangle \mathbf{e}_{1} \mathbf{e}_{2} \mathbf{e}_{3}$ in Euclidean meaning, hence Lemma 3.4 implies

$$
\mathbf{m}_{i}^{\mathcal{H}} \rightarrow \mathbf{m}_{i} \text { and } \mathbf{m}_{i}^{\mathcal{E}} \rightarrow \mathbf{m}_{i} \quad \text { for every } i \in\{1,2,3\} .
$$

On the other hand, for well-chosen $\mathbf{x}_{1}, \mathbf{x}_{2}, \mathbf{x}_{3}$, Theorems 4.2 and 4.4 imply that

$$
\mathbf{b}_{i}^{\mathcal{E}} \rightarrow \mathbf{b}_{i} \text { and } \mathbf{b}_{i}^{\mathcal{H}} \rightarrow \mathbf{b}_{i} \quad \text { for every } i \in\{1,2,3\}
$$

as $\varepsilon \rightarrow 0$.

By (5.3) and (5.4) the hyperbolic circumcenter $\mathbf{c}$ of the triangle $\triangle \mathbf{v}_{1} \mathbf{v}_{2} \mathbf{v}_{3}$ tends to the center $\mathbf{o}$ of $\mathcal{E}$ as $\varepsilon \rightarrow 0$, and therefore the circumcenter $\mathbf{c}$ is in the interior of the triangle $\triangle \mathbf{v}_{1} \mathbf{v}_{2} \mathbf{v}_{3}$ if $\varepsilon$ is small enough.

Suppose that the triangle $\triangle \mathbf{v}_{1} \mathbf{v}_{2} \mathbf{v}_{3}$ has also an $\mathcal{H}$-circumcenter, say $\mathbf{c}^{\prime}$. By (5.3) and (5.4) the $\mathcal{H}$-circumcenter $\mathbf{c}^{\prime}$ tends also to the center $\mathbf{o}$ of $\mathcal{E}$ as $\varepsilon \rightarrow 0$, hence it is in the interior of the triangle $\triangle \mathbf{v}_{1} \mathbf{v}_{2} \mathbf{v}_{3}$ if $\varepsilon$ is small enough.

On account of (5.1) and (5.2), for every $i \in\{1,2,3\}$ the closed segments $\overline{\mathbf{m}_{i}^{\mathcal{H}} \mathbf{b}_{i}^{\mathcal{H}}}$ and $\overline{\mathbf{m}_{i}^{\mathcal{E}} \mathbf{b}_{i}^{\mathcal{E}}}$ have $k(i) \geq 1$ points in common, which is on the same side of $\ell_{i}^{\prime}$ as $\mathbf{m}_{i}$ is. By the notice after the relations (5.1) and (5.2), we may assume that $k(2)=1$ 
and $k(3)=1$. This implies that $\mathbf{c}^{\prime}$ is in the left open half plane of the lines $\mathbf{m}_{i}^{\mathcal{E}} \mathbf{b}_{i}^{\mathcal{E}}$ directed from $\mathbf{m}_{i}^{\mathcal{E}}$ to $\mathbf{b}_{i}^{\mathcal{E}}$ for every $i \in\{2,3\}$ and it is in the left closed half plane of the lines $\mathbf{m}_{1}^{\mathcal{E}} \mathbf{b}_{1}^{\mathcal{E}}$ directed from $\mathbf{m}_{1}^{\mathcal{E}}$ to $\mathbf{b}_{1}^{\mathcal{E}}$. This contradicts the fact that the intersection of these half planes are empty, therefore the supposition of the existence of $\mathbf{c}^{\prime}$ was wrong, and the theorem is proved.

Existence of the orthocenter of a trigon, the common point of the three altitudes, is well known in Euclidean plane. It is also known for the hyperbolic plane [9, Theorem 3]: In hyperbolic geometry the altitudes of any trigon form a pencil.

Theorem 5.2. If the altitudes of any trigon in a Hilbert geometry $\mathcal{H}$ form a pencil, then $\left(\mathcal{H}, d_{\mathcal{H}}\right)$ is the hyperbolic geometry.

Proof. Following the proof of Theorem 5.1 we have the very same construction of the tangents and points, but without the midpoints for now.

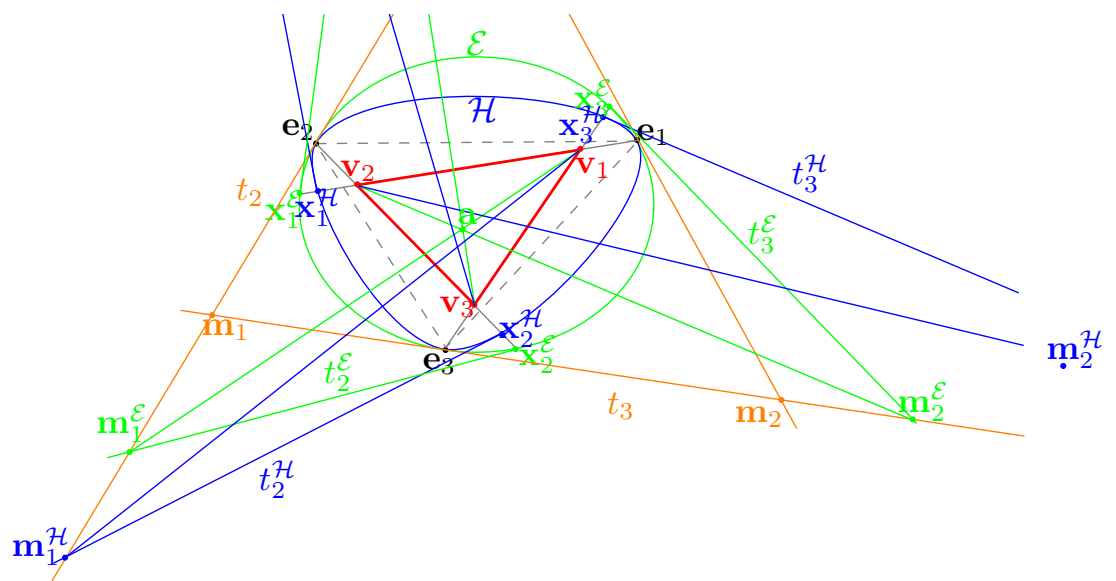

FIGURE 5.2. A triangle with altitudes intersecting in inner points

According to (5.3), the intersection $\mathbf{a}$ of the hyperbolic altitudes $\mathbf{v}_{i} \mathbf{m}_{i}^{\mathcal{E}}(i=$ $1,2,3)$ of the triangle $\triangle \mathbf{v}_{1} \mathbf{v}_{2} \mathbf{v}_{3}$ tends to the center $\mathbf{o}$ of $\mathcal{E}$ as $\varepsilon \rightarrow 0$, and therefore $\mathbf{a}$ is in the interior of the triangle $\triangle \mathbf{v}_{1} \mathbf{v}_{2} \mathbf{v}_{3}$ if $\varepsilon$ is small enough.

Suppose that the $\mathcal{H}$-altitudes $\mathbf{v}_{i} \mathbf{m}_{i}^{\mathcal{H}}(i=1,2,3)$ of the triangle $\triangle \mathbf{v}_{1} \mathbf{v}_{2} \mathbf{v}_{3}$ also intersect in a point $\mathbf{a}^{\prime}$.

By (5.3) the point $\mathbf{a}^{\prime}$ also tends to the center of $\mathcal{E}$ as $\varepsilon \rightarrow 0$, hence it is in the interior of the triangle $\triangle \mathbf{v}_{1} \mathbf{v}_{2} \mathbf{v}_{3}$ if $\varepsilon$ is small enough.

On account of relations $(5.2), \mathbf{v}_{i}=\mathbf{v}_{i} \mathbf{m}_{i}^{\mathcal{H}} \cap \mathbf{v}_{i} \mathbf{m}_{i}^{\mathcal{E}}$ for every $i \in\{1,2,3\}$, and therefore $\mathbf{a}^{\prime}$ is in the left open half plane of the lines $\mathbf{v}_{i} \mathbf{m}_{i}^{\mathcal{E}}$ directed from $\mathbf{v}_{i}$ to $\mathbf{m}_{i}^{\mathcal{E}}$ for every $i \in\{1,2,3\}$. This contradicts the fact that the intersection of these halfplanes are empty, therefore the supposition of the existence of $\mathbf{a}^{\prime}$ was wrong, and the theorem is proved. 
Acknowledgement. This research was supported by the European Union and co-funded by the European Social Fund under the project "Telemedicine-focused research activities on the field of Mathematics, Informatics and Medical sciences" of project number 'TÁMOP-4.2.2.A-11/1/KONV-2012-0073".

The authors appreciate János Kincses, Gábor Nagy, György Kiss and Tamás Szőnyi for their helpful discussions.

Thanks goes also to the anonymous referee whose advises improved this paper and in particular figures 5.1 and 5.2 considerable.

\section{REFERENCES}

[1] D. Amir, Characterizations of Inner Product Spaces, Birkhäuser Verlag, Basel - Boston Stuttgart, 1986.

[2] H. Busemann and P. J. Kelly, Projective Geometries and Projective Metrics, Academic Press, New York, 1953. v+332 pp.

[3] P. M. Gruber and F. E. Schuster, An arithmetic proof of Johns ellipsoid theorem, Arch. Math., 85(2005), 82-88.

[4] P. M. Gruber, Convex and Discrete Geometry, Springer-Verlag, Berlin - Heidelberg, 2007.

[5] R. Guo, Characterizations of hyperbolic geometry among Hilbert geometries: A survey, Handbook of Hilbert Geometry (eds.: A. Papadopoulos, A. Troyanov, IRMA Lectures in Mathematics and Theoretical Physics Vol. 22, European Mathematical Society Publishing House, 2014, doi: 10.4171/147), pp.: 147-158; doi: 10.4171/147-1/6; Also available at: http: //www.math. oregonstate.edu/ guore/docs/survey-Hilbert.pdf.

[6] D. Hilbert, Foundations of Geometry, Open Court Classics, Lasalle, Illinois, 1971.

[7] Á. G. Horváth, Semi-indefinite inner product and generalized Minkowski spaces, J. Geom. Phys., 60 (2010) 1190-1208.

[8] Á. G. Horváth, Premanifolds, Note di Math., 31/2 (2011), 17-51.

[9] N. Ivanov, V. Arnol'd, the Jacobi identity, and orthocenters, Amer. Math. Monthly, 118(2011), 41-65; doi: 10.4169/amer.math.monthly.118.01.041.

[10] P. J. Kelly and L. J. Paige, Symmetric Perpendicularity in Hilbert Geometries, Pacific J. Math., 2 (1952), 319-322.

[11] F. Kárteszi, Introduction to Finite Geometries, Disquisitiones Mathematicae Hungaricae 7, Akadémiai Kiadó, Budapest, 1976 (translated from the Hungarian version: Bevezetés a véges geometriákba, Akadémiai Kiadó, Budapest, 1972).

[12] Gy. Kiss and T. Szőnyi, Véges geometriák, Polygon, Szeged, 2001 (in Hungarian).

[13] J. Kozma and Á. Kurusa, Ceva's and Menelaus' Theorems characterize hyperbolic geometry among Hilbert geometries, J. Geom, (2014), to appear; doi: 10.1007/s00022-014-0258-7.

[14] G. E. Martin, The Foundations of Geometry and the Non-Euclidean Plane, Springer Verlag, New York, 1975.

[15] H. Martini, K. Swanepoel and G. Weiss, The geometry of Minkowski spaces - a survey. Part I, Expositiones Mathematicae, 19 (2001), 97-142.

[16] H. Martini and K. Swanepoel, The geometry of Minkowski spaces - a survey. Part II, Expositiones Mathematicae, 22(2) (2004), 93-144.

[17] B. Segre, Ovals in a finite projective plane, Canadian J. Math., 7 (1955), 414-416; doi: 10.4153/CJM-1955-045-x. 
József Kozma, Bolyai Institute, University of Szeged, Aradi vértanúk tere 1, 6725 Szeged (Hungary); E-mail: kozma@math.u-szeged.hu .

ÁRPÁD Kurusa, Bolyai Institute, University of Szeged, Aradi vértanúk tere 1, 6725 Szeged (Hungary); E-mail: kurusa@math.u-szeged.hu . 